\title{
Pathophysiological Mechanisms of Endogenous FVIII Release following Strenuous Exercise in Non-severe Haemophilia: A Review
}

\author{
C.L. Venema ${ }^{1}$ R.E.G. Schutgens ${ }^{2} \quad$ K. Fischer ${ }^{1}$ \\ ${ }^{1}$ Van Creveldkliniek, University Medical Centre Utrecht, Utrecht, \\ The Netherlands \\ ${ }^{2}$ Department of Hematology, University Medical Centre Utrecht, \\ Utrecht, The Netherlands
}

\begin{abstract}
Address for correspondence K. Fischer, MD, PhD, Van Creveldkliniek, University Medical Centre Utrecht, Heidelberglaan 100, 3584 CX Utrecht, The Netherlands (e-mail: k.fischer-2@umcutrecht.nl).
\end{abstract}

Thromb Haemost 2017;117:2237-2242.

\begin{abstract}
Introduction Non-severe haemophilia A is characterized by coagulation Factor VIII activity (FVIII:C) levels of 1 to $40 \mathrm{IU} / \mathrm{dL}$. It has been reported that strenuous exercise increases the plasma FVIII:C in haemophilia A patients. This review highlights current knowledge about the pathophysiological mechanisms of endogenous FVIII release following strenuous exercise.

Methods A literature search was performed to include relevant studies with data on pathophysiological mechanisms of FVIII release following strenuous exercise in haemophilia.

Results The source of the released FVIII is most likely endothelial cells (ECs) from different vascular beds. ECs from human lung, lymph, heart, intestine, skin and pulmonary artery can release and even produce FVIII in response to activation by

Keywords

- epinephrine

- exercise

- FVIII

- haemophilia

- endothelial cells epinephrine. Ex vivo evidence suggests that FVIII is co-stored with von Willebrand factor in Weibel-Palade bodies in some forms of non-severe haemophilia. The $\beta$-adrenergic receptor pathway is involved in increased FVIII levels following strenuous exercise.

Conclusion The current available ex vivo and in vivo evidence suggests that endogenous FVIII is released by ECs from different vascular beds in response to epinephrine following strenuous exercise in patients with non-severe haemophilia.
\end{abstract}

\section{Introduction}

Coagulation factor VIII (FVIII) is an essential protein co-factor in the intrinsic pathway of coagulation and plays an important role in secondary haemostasis. ${ }^{1}$ In plasma, FVIII can only circulate together with von Willebrand factor (vWF) that prevents premature clearance and proteolytic degradation, and thereby functions as carrier protein of FVIII. ${ }^{2}$ Normal plasma FVIII levels average approximately $100 \mathrm{IU} / \mathrm{dL}$, expressed as $100 \%$ plasma FVIII activity (FVIII:C). ${ }^{3}$ A genetic defect in expression or function of FVIII results in the recessive X-chromosome-linked bleeding disorder hae- mophilia A, affecting 1 in 5,000 males. ${ }^{1}$ In patients with the severe haemophilia phenotype, functional FVIII levels are undetectable (expressed as $<1 \%$ ). Moderate and mild haemophilia patients show functional FVIII levels of 1 to 5 and 6 to $40 \%$, respectively. ${ }^{4}$ These patients can be treated with vasopressin analogue desmopressin (DDAVP) infusions, which induce a temporary rise in plasma FVIII:C and vWF levels ${ }^{5}$ and can be used for treatment during bleeding or surgical procedures. ${ }^{1}$ Interestingly, it is demonstrated that physical exercise also increases plasma FVIII levels in these patients. ${ }^{3}$ The information on the complex mechanisms in release of additional endogenous FVIII during exercise 
is rapidly increasing. This review summarizes current evidence regarding the pathophysiological mechanisms of endogenous FVIII release following strenuous exercise in haemophilia.

\section{Methods}

A systemic literature search was performed of studies published from 1990 until 7 July 2016 using the electronic PubMed (http://www.ncbi.nlm.nih.gov/sites/entrez/) and EMBASE (http://www.embase.com) databases. The following search terms and synonyms of these search terms were used: adrenergic nervous system, blood coagulation, DDAVP, epinephrine, exercise, FVIII:C, haemophilia and Weibel-Palade Bodies. This search was run on 15 January and 7 July 2016. Titles and abstracts of articles were screened to identify potentially relevant studies by one researcher. These studies were recruited as complete manuscript and relevant studies in English were used for this review article.

\section{FVIII Production and Storage}

\section{FVIII Production by Endothelial Cells}

The simple question 'Where is FVIII made?' has been studied for decades. Although many contradictory reports about specific FVIII-producing cell and tissue types have been published, major evidence points to FVIII production by different endothelial cell (EC) populations in vivo. ${ }^{6}$ The liver was thought to be the primary source of FVIII based on the fact that liver transplantation re-establishes circulating FVIII levels in haemophilia A patients. ${ }^{7}$ However, the specific cell type within the organ producing FVIII has been debated for many years. The observation that a haemophilic phenotype is not observed after liver transplantation from haemophilic donors into non-haemophilic recipients indicates that there are extrahepatic sites that significantly contribute to FVIII biosynthesis. ${ }^{8}$ This is corroborated by the observation that plasma FVIII levels are normal or even elevated during severe liver diseases when low plasma levels of the other coagulation factors are found. ${ }^{6}$ Everett et al confirmed this by knocking out the cargo receptor lectin mannose-binding 1 (LMAN1) in several murine tissues. LMAN1 is essential in the secretory pathway of FVIII to the plasma and mutations in this receptor result in deficiency of FVIII with levels reduced to 10 to $15 \%$ of normal human plasma. In this experiment, plasma FVIII levels did not differ between hepatocyte-specific LMAN1 knockout and control mice, whereas FVIII activity levels were markedly decreased in endothelial-specific LMAN1 knockout mice. This indicates that hepatocytes do not contribute significantly to the plasma FVIII pool in vivo. ${ }^{9}$ Fahs et al also observed severe haemophilia phenotypes in endothelial FVIII-knockout models without detectable plasma FVIII: $\mathrm{C}$ in chromogenic assays, indicating ECs as the predominant source of plasma FVIII. ${ }^{10}$ Possibly, hepatocytes may contribute to steady-state concentrations of circulating FVIII, but ECs are most likely important in acute release of FVIII. This hypothesis may be studied in patients who received liver-targeted gene therapy ${ }^{11}$ : after successful gene therapy, these patients may show stable levels of FVIII, but would be unable to release FVIII in response to DDAVP or other adrenaline-receptor stimulation.

FVIII Production by ECs of Different Vascular Beds Microscopic studies demonstrated FVIII to be largely produced by liver sinusoidal endothelial cells (LSECs), ${ }^{9}, 10$ while FVIII mRNA (messenger ribonucleic acid) was also detected in ECs of the kidneys, spleen and lungs. In contrast, the brain and heart did not express FVIII mRNA. ${ }^{9,12,13}$ Shahani et al showed that the microvascular ECs from lung, heart, intestine, skin and pulmonary artery secrete FVIII and release it after stimulation with endothelial agonists in vitro. In contrast, there is no evidence of FVIII synthesis in larger vessels since FVIII release was not detected in ECs from the aorta, umbilical artery and umbilical vein. ${ }^{6}$ FVIII secretion by human adult pulmonary microvascular ECs (HPMECs) was also established ex vivo and in vitro by Jacquemin et al. ${ }^{14}$ This indicates the important role of HPMECs as a source of FVIII, through either synthesis or local storage. Interestingly, Pan et al also recently published data about lymphatic ECs (LECs) as extrahepatic FVIII source in vivo. FVIII and vWF were shown to be co-expressed in post-capillary high endothelial venules. Moreover, FVIII was secreted by LECs and LSECs in vitro and FVIII:C was determined in both mouse and human lymph by using FVIII:C assays. ${ }^{15}$ This suggests LSECs, LECs and HPMECs as potential source of FVIII release to regulate FVIII release. Altogether, there is convincing evidence that ECs of different vascular beds produce FVIII in vitro and in vivo.

\section{FVIII Co-storage in Endothelial Weibel-Palade Bodies} Extrahepatic ECs from different vascular beds seem to be important both in FVIII production and in storage of FVIII. ${ }^{6}$ The average plasma level of FVIII is $100 \mathrm{IU} / \mathrm{dL}$ and its half-life is 10 to 12 hours. Since infusion of the endothelial agonist epinephrine increases FVIII plasma levels by two to three times within only 10 minutes, this rapid rise indicates FVIII secretion from a storage pool rather than novo protein synthesis. A similar rise in vWF plasma levels is observed in vivo, suggesting a co-storage pool for FVIII and vWF in ECs that are released upon stimulation with epinephrine. This was confirmed by immunofluorescent staining for FVIII and vWF in microscopic studies that showed localization of FVIII in vesicles of human cardiac microvascular ECs, human umbilical vein ECs (HUVECs) and human glomerular microvascular ECs (GMVECs). These GMVECs were also strongly stained with vWF, suggesting co-storage in Weibel-Palade bodies (WPBs) of ECs. ${ }^{6,16,17}$ Stimulation of HUVECs and GMVECs by endothelial agonists resulted in simultaneous release of functionally active FVIII and vWF proteins most likely by exocytosis of these WPBs, ${ }^{16,17}$ indicating the importance of endothelial WPBs in FVIII release.

Whether FVIII and vWF are synthesized endogenously in one cell type was studied by Haberichter et al. ECs were able to take up FVIII neither from the culture nor via cell-cell contact, indicating vascular endothelium as the location of a 
releasable storage pool of endogenously synthesized FVIII. ${ }^{18}$ It has been shown that targeting of FVIII to WPBs by using plasmid mutagenesis can even restore haemostasis in haemophilia A mice, supporting the potential role of WPBs in FVIII release upon stimulation. ${ }^{16}$

In conclusion, WPBs of extrahepatic ECs from different vascular beds seem to be important in regulating FVIII secretion. $^{6}$

\section{FVIII Release upon Activation of the Adrenergic Nervous System}

In humans, increased plasma FVIII:C is observed during circumstances of physical stress, such as pregnancy, surgery, chronic inflammation, malignancy, liver disease, hyperthyroidism, intravascular haemolysis and renal disease. ${ }^{19}$ Both in vitro and in vivo studies have provided evidence supporting the hypothesis that elevation of plasma FVIII levels during circumstances of physical stress is a result of activation of the $\beta$-adrenergic receptor pathway.

\section{FVIII Release by WPBs in Response to Epinephrine}

Endothelial WPBs are determined as a source of FVIII and vWF upon stimulation with epinephrine in both in vitro and in vivo studies. In the study of Van den Biggelaar et al, human blood outgrowth ECs (BOECs), ECs obtained from healthy donor peripheral blood samples and cultured, were transduced with a lentiviral vector encoding FVIII. After stimulation with epinephrine, FVIII was released together with vWF. $^{20}$ The effect of epinephrine on FVIII release by microvascular ECs was demonstrated in vitro by Shahani et al. ECs from lung, heart, intestine, skin and pulmonary artery were cultured and stimulated by epinephrine, resulting in FVIII release. $^{6}$

In vivo FVIII release by ECs after stimulation with epinephrine was determined by Shi et al. ${ }^{21}$ Transgenic T2F8 $\left(\mathrm{T} 2 \mathrm{~F}^{\mathrm{Tg}}\right)$ mice, expressing human B-domain-deleted FVIII under the control of EC-specific promoter of the Tie2 receptor tyrosine kinase gene, were subcutaneously injected with epinephrine ( $2 \mathrm{mg} / \mathrm{kg}$ body weight). FVIII:C and von Willebrand factor antigen (vWF:Ag) increased 1.68 and 2.08 times, respectively, in plasma from $\mathrm{T}_{2} \mathrm{~F}^{\mathrm{Tg}}$ mice, whereas this was increased 1.27 and 2.21 times, respectively, in wild-type mice after administration of epinephrine. This demonstrates ECs act as storage pool for FVIII, which is released in response to epinephrine, mimicking physical stress responses in vivo. ${ }^{21}$
However, the downstream signalling pathway of epinephrine in ECs remains largely unknown. It has only been shown that after binding to the cell surface receptor, epinephrine increases cyclic adenosine monophosphate (cAMP) levels within the cell to fine-tune endothelial responses. ${ }^{22}$ But how this cAMP-dependent signalling leads to exocytosis of WPBs, resulting in increased plasma FVIII concentrations, remains unknown. Further research should be done to downstream effects of cAMP to stimulate FVIII secretion and thereby to restore haemostasis in non-severe haemophilia patients.

\section{Blocking of $\beta$-Receptor Pathway Blunts FVIII Release} Decades ago, it was already confirmed that intravenous infusions of propranolol, a blocker of the $\beta$-receptor pathway, blunts the increasing effect of epinephrine on plasma FVIII levels. ${ }^{23}$ Adult subjects, selected on clearly rising plasma FVIII levels after infusion with epinephrine, were intravenously injected with epinephrine and with either the $\alpha$-blocker phentolamine, or one of the two $\beta$-blockers pronethalol and propranolol preceding epinephrine infusion.

The effect of epinephrine on plasma FVIII levels was reduced after administering phentolamine, although this difference was not significant. In contrast, $\beta$-blocker administration did result in significantly lowered plasma FVIII levels compared with saline treatment and the values were similar in response to pronethalol and propranolol. ${ }^{23}$ Furthermore, a therapeutic use of this phenomenon was published. Hoppener et al showed a $23 \%$ decrease in FVIII:C $(p<0.0001)$ after 14 days of treatment with the $\beta$-receptor blocker propranolol in patients with deep vein thrombosis. ${ }^{24}$ Ten weeks after discontinuing of propranolol, a return of FVIII to its original high level was found. Thus, elevated plasma FVIII levels can be decreased by blocking the $\beta$ receptor, ${ }^{24}$ supporting the relation between plasma FVIII:C and the adrenergic nervous system (-Table $\mathbf{1}$ ).

\section{FVIII Storage in Non-severe Haemophilia}

There is ex vivo evidence that the co-storage of FVIII with vWF is independent of the capacity of FVIII to bind to $\mathrm{vWF}^{25}$ To determine co-storage of FVIII with vWF in non-severe haemophilia due to mutations associated with reduced binding with vWF, Van den Biggelaar et $\mathrm{al}^{20}$ studied intracellular co-trafficking in human embryonic kidney cells and BOECs. The isolated and cultured ECs were transduced with lentiviral factors encoding FVIII mutations associated with mild or moderate haemophilia A due to reduced binding to vWF. They

Table 1 The effect of the $\beta$-adrenergic nervous system on FVIII:C release by endothelial cells

\begin{tabular}{|l|l|l|l|l|l|}
\hline Study & N & Model & Agonist & Antagonist & Relative change FVIII:C \\
\hline Shi et $\mathrm{al}^{21}$ & 4 & WT mice & Epinephrine & & 1.27 \\
\hline Shi et $\mathrm{al}^{21}$ & 7 & T2F8 & & 1.68 \\
\hline Ingram et $\mathrm{al}^{23}$ & 9 & Human & Epinephrine & & -1.06 \\
\hline Hoppener et al & & & Propranolol & -4.35 \\
\hline
\end{tabular}

Abbreviation: DVT, deep vein thrombosis; WT, wild type; $T 2 \mathrm{~F}^{\mathrm{Tg}}$, transgenic T2F8.

Note: ECs were either stimulated with the agonist epinephrine or suppressed by the antagonist propranolol. 
demonstrated expression of all mutated FVIII variants within vWF-containing storage organelles despite impaired binding of FVIII with vWF. According to the authors, these data support the hypothesis that in vivo WPBs represent a storage pool of vWF and FVIII that can be released upon DDAVP administration in non-severe haemophilia patients. ${ }^{25}$

\section{Endothelial-Derived FVIII corrects haemophilia A}

\section{Correction of Haemophilia Phenotype in FVIII Knockout Mice}

Interestingly, in vivo mouse models established correction of the haemophilia A phenotype by endothelial-derived FVIII. The ability to form a clot and to survive tail clipping was studied in FVIII knockout mice (FVIII ${ }^{\text {null }}$ ) and FVIII ${ }^{\text {null }}$ mice expressing human B-deleted FVIII in the vascular endothelium (FVIII ${ }^{\text {null_ECFVIII }}$ ). This FVIII from endothelial origin was able to normalize in vivo clotting: in contrast to control

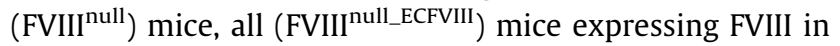
the vascular endothelium survived tail clipping similar to wild-type mice. Furthermore, the arterial thrombosis model showed stable clot formation in FVIII ${ }^{\text {null_ECFVIII }}$ mice, rescuing them from bleeding, whereas clot formation was absent in FVIII $^{\text {null }}$ mice. In the venous thrombosis model, FVIII ${ }^{\text {null_ECFVIII }}$ mice displayed clot volumes 23 times higher in comparison with FVIII ${ }^{\text {null }}$ mice $(p<0.05)$. Moreover, the clot volume was similar to clot volume in wild-type mice. Thus, expression of B-deleted human FVIII in the vascular endothelium corrects the haemophilic A phenotype, ${ }^{21}$ supporting the potential therapeutic role of endothelial-derived FVIII in restoring haemostasis in non-severe haemophilia patients.

\section{Correction Haemophilia Phenotype Patients}

It is hypothesized that the pathophysiologic mechanism of endogenous FVIII release following strenuous exercise is similar to FVIII release following DDAVP administration. The hormone DDAVP acts as agonist on the vasopressin V2 receptor of ECs, leading to a rise in intracellular levels of the second messenger cAMP that induces exocytosis of WPBs. ${ }^{26}$ Intravenous or intranasal administration of DDAVP to healthy individuals leads to increased plasma FVIII:C and vWF levels, making it an accepted therapy for mild haemophilia patients. ${ }^{27}$ However, the response to DDAVP with regard to FVIII levels is variable within haemophilia patients due to different F8 gene mutations, with strongest effect in patients showing other amino acid changes than Arg2169His or Pro149Arg. ${ }^{28}$ In nephrogenic diabetes insipidus patients, who lack the V2 receptor, this rise in FVIII and vWF levels is not observed, ${ }^{29}$ indicating the important mechanism of endothelial-mediated FVIII release after DDAVP administration.

\section{Exercise and FVIII Release}

FVIII Release in Healthy Individuals

In healthy individuals, it is well established that elevations of FVIII:C occur following acute exercise and induce hypercoagulability $^{30}$ and that this activity remains increased during exercise recovery. ${ }^{31}$ Hypercoagulability following high-inten- sity exercise is explained by elevated catecholamine levels. ${ }^{32}$ Healthy young men (20-29 years) performed strenuous exercise (130\% of individual anaerobic intensities [IAT]) and moderate exercise ( $90 \%$ of IAT) by bicycle ergometer for 30 minutes. The IAT are described as estimation of the maximum lactate steady state of the participant, ${ }^{33}$ which is determined by maximal oxygen uptake or maximal heart rate corresponding to control exercise intensities. ${ }^{34}$ Menzel et al performed bicycle ergometer exercise tests of different intensities in 25 healthy, untrained, non-smokers. Coagulation was measured under flow condition, using full blood. Moderate exercise resulted in a slight but significant elevation of catecholamine levels, whereas strenuous exercise resulted in a more pronounced elevation. Concomitantly, FVIII levels were increased by $34 \%$ following moderate exercise compared with an increase of $63 \%$ after strenuous exercise. ${ }^{35}$

\section{FVIII Release in Haemophilia Patients}

The effect of exercise on plasma FVIII:C levels in haemophilia patients was determined in several studies reporting on small case series (-Table 2 ). All studies used one-stage laboratory assays and a bicycle ergometer or recumbent cross-trainer ${ }^{36}$ to induce the exercise. One study reported on very mild aquatic exercise, without defining intensity. ${ }^{37}$ Moderate exercise intensity was defined as an intensity of 50 to $65 \%$ of maximum heart rate. Strenuous exercise was defined as exercise until maximum heart rate and/or maximum oxygen consumption ${ }^{3}$ or reaching $85 \%$ of maximum heart rate. ${ }^{38}$ Several trends can be observed in this table. First, the response of FVIII is dependent on the intensity of exercise, showing a higher increase after strenuous exercise similar to non-haemophilia patients. Second, the effects of exercise on coagulation are most pronounced within $10 \mathrm{~min}-$ utes after exercise, so the timing of assessment should be taken into account when comparing studies.

The increase of FVIII is proportional and therefore dependent on baseline FVIII. An increase in FVIII levels of patients with severe haemophilia was not detected in the seven severe patients reported by Koch et $\mathrm{al}^{39}$ nor in the 15 patients (all with intron 22 inversion) studied by Zourikian et $\mathrm{al}^{40}$ or the six studied by Kumar et al. ${ }^{38}$ However, Li et al were able to measure levels $<1.00 \mathrm{IU} / \mathrm{dL}$ and did show a 1.41 fold increase in FVIII levels after moderate exercise. ${ }^{36}$ Strikingly, 4 of 11 patients even achieved a level of $1.00 \mathrm{IU} / \mathrm{dL}$ or higher following exercise. However, in these patients with severe haemophilia, vWF:Ag did increase by $48 \%,{ }^{40}$ similar to the increase observed in non-severe haemophilia patients studied by Groen et al. ${ }^{3}$

Two studies reported increase of FVIII following strenuous exercise in non-severe haemophilia. The increase in FVIII appeared higher in the study by Groen et al than in the study by Kumar et al. However, this may partly be explained by the difference in age. Importantly, Kumar et al observed that the response to exercise appeared age dependent, for both FVIII and vWF:Ag; only patients aged 13 years or older $(n=8)$ showed a significant increase in FVIII (mean relative increase of 2.34) and vWF-Ag (mean relative increase of 1.56) 5 minutes after exercise. ${ }^{38}$ And these values are very close to the 
Table 2 Studies reporting on the effect of exercise on FVIII and VWF antigen (VWF:Ag) levels in haemophilia A patients

\begin{tabular}{|c|c|c|c|c|c|c|c|}
\hline Study & $N$ & $\begin{array}{l}\text { Age (y), } \\
\text { mean (range) }\end{array}$ & $\begin{array}{l}\text { Baseline } \\
\text { FVIII (IU/dL), } \\
\text { mean (range) }\end{array}$ & $\begin{array}{l}\text { Exercise } \\
\text { intensity }\end{array}$ & $\begin{array}{l}\text { sampling } \\
\text { time post } \\
\text { exercise } \\
\text { (min) }\end{array}$ & $\begin{array}{l}\text { Relative } \\
\text { increase in } \\
\text { FVIII:C, } \\
\text { mean (range) }\end{array}$ & $\begin{array}{l}\text { Relative } \\
\text { increase in } \\
\text { vWF:Ag, } \\
\text { mean (range) }\end{array}$ \\
\hline \multicolumn{8}{|l|}{ Severe haemophilia } \\
\hline Beltrame et al $\left.\right|^{37}$ & 5 & N.R. $(15-34)$ & N.R. & Very light & $<1$ & 1.0 & N.R. \\
\hline Li et $\mathrm{al}^{36}$ & 11 & $12.7(6-15)$ & $0.7(0.5-0.8)$ & Moderate & $<1$ & 1.41 & N.R. \\
\hline Koch et al ${ }^{39}$ & 7 & $11.6(8.3-15.5)$ & $<1.0$ & Moderate & 10 & 1.0 & N.R. \\
\hline Zourikian et al ${ }^{40}$ & 15 & $23.5(19-21)^{a}$ & $<1$ & Moderate & 60 & 1.0 & 1.48 \\
\hline Kumar et al ${ }^{38}$ & 6 & $14.2(5-18)$ & $<1.0$ & Strenuous & 5 & 1.0 & 1.3 \\
\hline Kumar et al $\left.\right|^{38}$ & 6 & $14.2(5-18)$ & $<1.0$ & Strenuous & 60 & 1.0 & 1.2 \\
\hline \multicolumn{8}{|c|}{ Mild-moderate haemophilia } \\
\hline Beltrame et $\mathrm{al}^{37}$ & 5 & N.R. (15-34) & N.R. & Very light & $<1$ & 1.7 & N.R. \\
\hline Ravanbod et al ${ }^{41}$ & 10 & $24.5(17-38)$ & 12 (N.R.) & Moderate & 10 & 1.3 & N.R. \\
\hline Kumar et al $\left.\right|^{38}$ & 13 & $12.8(5-18)$ & $8(5-10)$ & Strenuous & 5 & 1.9 & 1.4 \\
\hline Groen et al $\left.\right|^{3}$ & 15 & $27(20-39)^{a}$ & $5(2-15)^{a}$ & Strenuous & 10 & $2.5(1.8-7.0)$ & $1.5(1.08-2.23)$ \\
\hline Kumar et al $\left.\right|^{38}$ & 13 & $12.8(5-18)$ & $8(5-10)$ & Strenuous & 60 & 1.5 & 1.1 \\
\hline Groen et $\mathrm{al}^{3}$ & 7 & N.R. & N.R. & DDAVP & 60 & 3.5 & N.R. \\
\hline
\end{tabular}

Abbreviations: DDAVP, desmopressin; N.R., not reported.

${ }^{\mathrm{a}}$ Median values given.

observations by Groen et al in patients with a minimum age of 20 years. $^{3}$

Although the response to exercise is related to FVIII:C levels, intensity and age, it is complicated to determine how much therapeutic potential exercise has in different patients. The FVIII response to exercise is known to be stronger in healthy adults of younger age with higher cardiorespiratory activity levels ${ }^{42}$ and activation of the coagulation system is less pronounced in athletes compared with untrained individuals. ${ }^{43}$ More studies are needed to establish the practical consequences of changes in coagulation following exercise in patients with haemophilia.

\section{Similar Increase FVIII:C by DDAVP}

To compare the effect of strenuous exercise on increased plasma FVIII:C with administration of DDAVP, results of DDAVP response tests in mild haemophilia patients were compared with FVIII:C after strenuous exercise in one study and it showed relative elevated plasma FVIII:C levels of 3.5 times (from 6 to $40 \mathrm{IU} / \mathrm{dL}$ ) after DDAVP administration. This increase was similar to the increase in plasma FVIII:C after strenuous exercise $(p=0.14),{ }^{3}$ supporting strenuous exercise as potential therapy to increase endogenous FVIII release in mild haemophilia patients. It is suggested that both DDAVP and exercise target the same signalling pathway in the same cell type and are thereby part of the same system that releases additional endogenous FVIII. ${ }^{26}$

\section{Conclusion}

FVIII can be released from ECs in different vascular beds in response to activation by epinephrine. Ex vivo evidence suggests that non-severe haemophilia FVIII variants are costored with VWF in WPBs of ECs. There is evidence that both exercise and DDVAP increase circulating FVIII concentrations by acting on CAMP-dependent signalling in ECs resulting in exocytosis of FVIII-containing WPBs. This suggests that exercise and DDAVP are part of the same pathophysiologic mechanism. It should be further investigated if performing exercise at optimal intensity does reduce bleeding complications during physical activity in non-severe haemophilia A.

\section{References}

1 Spiegel PC Jr, Stoddard BL. Optimization of factor VIII replacement therapy: can structural studies help in evading antibody inhibitors? Br J Haematol 2002;119(02):310-322

2 Lenting PJ, van Mourik JA, Mertens K. The life cycle of coagulation factor VIII in view of its structure and function. Blood 1998; 92(11):3983-3996

3 Groen WG, den Uijl IE, van der Net J, Grobbee DE, de Groot PG, Fischer K. Protected by nature? Effects of strenuous physical exercise on FVIII activity in moderate and mild haemophilia A patients: a pilot study. Haemophilia 2013;19(04):519-523

4 Mannucci PM, Tuddenham EG. The hemophilias: from royal genes to gene therapy. N Engl J Med 2001;344(23):1773-1779

5 Lethagen S. Desmopressin in mild hemophilia A: indications, limitations, efficacy, and safety. Semin Thromb Hemost 2003; 29(01):101-106

6 Shahani T, Lavend'homme R, Luttun A, Saint-Remy JM, Peerlinck $\mathrm{K}$, Jacquemin $\mathrm{M}$. Activation of human endothelial cells from specific vascular beds induces the release of a FVIII storage pool. Blood 2010;115(23):4902-4909

7 Bontempo FA, Lewis JH, Gorenc TJ, et al. Liver transplantation in hemophilia A. Blood 1987;69(06):1721-1724

8 Madeira CL, Layman ME, de Vera RE, Fontes PA, Ragni MV. Extrahepatic factor VIII production in transplant recipient of hemophilia donor liver. Blood 2009;113(21):5364-5365 
9 Everett LA, Cleuren AC, Khoriaty RN, Ginsburg D. Murine coagulation factor VIII is synthesized in endothelial cells. Blood 2014;123 (24):3697-3705

10 Fahs SA, Hille MT, Shi Q, Weiler H, Montgomery RR. A conditional knockout mouse model reveals endothelial cells as the principal and possibly exclusive source of plasma factor VIII. Blood 2014; 123(24):3706-3713

11 Spencer HT, Riley BE, Doering CB. State of the art: gene therapy of haemophilia. Haemophilia 2016;22(Suppl 5):66-71

12 Aronovich A, Tchorsh D, Katchman H, et al. Correction of hemophilia as a proof of concept for treatment of monogenic diseases by fetal spleen transplantation. Proc Natl Acad Sci U S A 2006;103 (50):19075-19080

13 Kennedy A, Ng CT, Biniecka M, et al. Angiogenesis and blood vessel stability in inflammatory arthritis. Arthritis Rheum 2010;62(03): 711-721

14 Jacquemin M, Neyrinck A, Hermanns MI, et al. FVIII production by human lung microvascular endothelial cells. Blood 2006;108(02): 515-517

15 Pan J, Dinh TT, Rajaraman A, et al. Patterns of expression of factor VIII and von Willebrand factor by endothelial cell subsets in vivo. Blood 2016;128(01):104-109

16 Rosenberg JB, Greengard JS, Montgomery RR. Genetic induction of a releasable pool of factor VIII in human endothelial cells. Arterioscler Thromb Vasc Biol 2000;20(12):2689-2695

17 Turner NA, Moake JL. Factor VIII is synthesized in human endothelial cells, packaged in Weibel-Palade bodies and secreted bound to ULVWF strings. PLoS One 2015;10(10):e0140740

18 Haberichter SL, Shi Q, Montgomery RR. The biology of von Willebrand factor and factor VIII-regulated release. Haematol Rep 2005; 1:9-14

19 Bloom AL. The biosynthesis of factor VIII. Clin Haematol 1979; $8(01): 53-77$

20 van den Biggelaar M, Bouwens EA, Kootstra NA, Hebbel RP, Voorberg J, Mertens K. Storage and regulated secretion of factor VIII in blood outgrowth endothelial cells. Haematologica 2009; 94(05):670-678

21 Shi Q Fahs SA, Kuether EL, Cooley BC, Weiler H, Montgomery RR. Targeting FVIII expression to endothelial cells regenerates a releasable pool of FVIII and restores hemostasis in a mouse model of hemophilia A. Blood 2010;116(16):3049-3057

22 Vischer UM, Wollheim CB. Epinephrine induces von Willebrand factor release from cultured endothelial cells: involvement of cyclic AMP-dependent signalling in exocytosis. Thromb Haemost 1997;77(06):1182-1188

23 Ingram GI, Jones RV. The rise in clotting factor 8 induced in man by adrenaline: effect of alpha- and beta-blockers. J Physiol 1966; 187(02):447-454

24 Hoppener MR, Kraaijenhagen RA, Hutten BA, Büller HR, Peters RJ, Levi M. Beta-receptor blockade decreases elevated plasma levels of factor VIII:C in patients with deep vein thrombosis. J Thromb Haemost 2004;2(08):1316-1320

25 van den Biggelaar M, Bouwens EAM, Voorberg J, Mertens K. Storage of factor VIII variants with impaired von Willebrand factor binding in Weibel-Palade bodies in endothelial cells. PLoS One 2011;6(08):e24163

26 Mannucci PM. Desmopressin (DDAVP) in the treatment of bleeding disorders: the first 20 years. Blood 1997;90(07):2515-2521
27 Warrier AI, Lusher JM. DDAVP: a useful alternative to blood components in moderate hemophilia $A$ and von Willebrand disease. J Pediatr 1983;102(02):228-233

28 Stoof SC, Sanders YV, Petrij F, et al. Response to desmopressin is strongly dependent on F8 gene mutation type in mild and moderate haemophilia A. Thromb Haemost 2013;109(03): 440-449

29 Mannucci PM. Hemostatic drugs. N Engl J Med 1998;339(04): 245-253

30 Arai M, Yorifuji H, Ikematsu S, et al. Influences of strenuous exercise (triathlon) on blood coagulation and fibrinolytic system. Thromb Res 1990;57(03):465-471

31 Lin X, El-Sayed MS, Waterhouse J, Reilly T. Activation and disturbance of blood haemostasis following strenuous physical exercise. Int J Sports Med 1999;20(03):149-153

32 Ikarugi H, Shibata M, Shibata S, Ishii H, Taka T, Yamamoto J. High intensity exercise enhances platelet reactivity to shear stress and coagulation during and after exercise. Pathophysiol Haemost Thromb 2003;33(03):127-133

33 Urhausen A, Coen B, Weiler B, Kindermann W. Individual anaerobic threshold and maximum lactate steady state. Int J Sports Med 1993;14(03):134-139

34 Meyer T, Gabriel HH, Kindermann W. Is determination of exercise intensities as percentages of VO2max or HRmax adequate? Med Sci Sports Exerc 1999;31(09):1342-1345

35 Menzel K, Hilberg T. Blood coagulation and fibrinolysis in healthy, untrained subjects: effects of different exercise intensities controlled by individual anaerobic threshold. Eur J Appl Physiol 2011; 111(02):253-260

36 Li KX, Xiao J, Zhao YQ et al. Moderate-intensity exercise improves the thromboelastography coagulation index in children with severe hemophilia A. Blood Coagul Fibrinolysis 2016;27(07): 797-803

37 Beltrame LG, Abreu L, Almeida J, Boullosa DA. The acute effect of moderate intensity aquatic exercise on coagulation factors in haemophiliacs. Clin Physiol Funct Imaging 2015;35(03):191-196

38 Kumar R, Bouskill V, Schneiderman JE, et al. Impact of aerobic exercise on haemostatic indices in paediatric patients with haemophilia. Thromb Haemost 2016;115(06):1120-1128

39 Koch B, Luban NL, Galioto FM Jr, Rick ME, Goldstein D, Kelleher JF Jr. Changes in coagulation parameters with exercise in patients with classic hemophilia. Am J Hematol 1984;16(03):227-233

40 Zourikian N, Merlen C, Bonnefoy A, St-Louis J, Rivard GE. Effects of moderate-intensity physical exercise on pharmacokinetics of factor VIII and von Willebrand factor in young adults with severe haemophilia A: a pilot study. Haemophilia 2016;22(03):e177-e183

41 Ravanbod R, Torkaman G, Baghaipour MR, et al. Effects of ergometric exercise on F-VIII coagulant activity in mild and moderate haemophilia A: a chance to reduce injective replacement therapy. Pak J Biol Sci 2006;9:2823-2827

42 van den Burg PJ, Hospers JE, Mosterd WL, Bouma BN, Huisveld IA. Aging, physical conditioning, and exercise-induced changes in hemostatic factors and reaction products. J Appl Physiol (1985) 2000;88(05):1558-1564

43 Mandalaki T, Dessypris A, Louizou C, Panayotopoulou C, Dimitriadou C. Marathon Run III: effects on coagulation, fibrinolysis, platelet aggregation and serum cortisol levels. A 3-year study. Thromb Haemost 1980;43(01):49-52 